(2) Open Access Full Text Article

\title{
Cranberry syrup vs trimethoprim in the prophylaxis of recurrent urinary tract infections among children: a controlled trial
}

This article was published in the following Dove Press journal:

Open Access Journal of Clinical Trials

27 May 2012

Number of times this article has been viewed

Jose Uberos'

Mercedes Nogueras-Ocana ${ }^{2}$

Verónica Fernandez-

Puentes'

Rocio Rodriguez-Belmonte'

Eduardo Narbona-López'

Antonio Molina-Carballo'

Antonio Munoz-Hoyos'

'Paediatric Clinical Management Unit, San Cecilio University Clinical Hospital, Avda de Madrid s/n, Granada, Spain; ${ }^{2}$ Paediatric Urology, San Cecilio University Clinical Hospital, Avda de Madrid s/n, Granada, Spain
Correspondence: Jose Uberos Paediatric Clinical Management Unit. San Cecilio University Clinical Hospital, Avda de Madrid s/n, Granada, Spain Phone +3495802 34II

Fax +3495824666I

Email juberos@ugr.es
Objectives: The present study forms part of the ISRCTN16968287 clinical assay. The objective of this study was to determine the effectiveness of cranberry syrup in the prophylaxis of recurrent urinary tract infection (UTI).

Design: Phase III randomized clinical trial.

Setting: The study was conducted at the San Cecilio Clinical Hospital (Granada, Spain).

Participants: A total of 192 patients were recruited. The subjects were aged between 1 month and 13 years. Criteria for inclusion were a background of recurrent UTI (more than two episodes of infection in the last 6 months), associated or otherwise with vesicoureteral reflux of any degree, or renal pelvic dilatation associated with UTI. Criteria for exclusion from recruitment to the study included the co-existence of UTI with other infectious diseases or with metabolic diseases, chronic renal insufficiency, and the presence of allergy or intolerance to any of the components of cranberry syrup or trimethoprim.

Primary outcome measures: The primary objective was to determine the risk of UTI associated with each intervention.

Results: Of the 198 patients initially eligible, 192 were finally included in the study to receive either cranberry syrup or trimethoprim. UTI was observed in 47 patients, 17 of whom were males and 30 females. We recruited 95 patients diagnosed with recurrent UTI on entry; during follow-up, 26 patients had a UTI $(27.4 \%$, 95\% CI: $18.4 \%-36.3 \%)$. Six patients $(6.3 \%)$ were male and $20(21.1 \%)$ were female. Eighteen patients $(18.9 \%$ of the total, $95 \% \mathrm{CI}$ : $11 \%-26.3 \%)$ receiving trimethoprim had a UTI and eight patients $(8.4 \%$ of the total, $95 \%$ CI: $2.8 \%-13.9 \%$ ) were given cranberry. Sixty-six percent of the episodes of UTI recurrence were caused by Escherichia coli, with no significant differences being found between the two treatment branches. No differences were observed between the two treatment branches in the rate of resistance to antibiotics.

Conclusion: Our study confirms that cranberry syrup is a safe treatment for the pediatric population. Cranberry prophylaxis has noninferiority with respect to trimethoprim in recurrent UTI. (European Clinical Trials Registry EuDract 2007-004397-62) (ISRCTN16968287).

Keywords: cranberry, urinary tract infections, trimethoprim, vesicoureteral reflux, antibiotic prophylaxis

\section{Introduction}

Although there is some controversy, current evidence supports the use of long-term, low-dose antibiotics to control recurrent urinary tract infections (UTI), the number needed to treat (NNT) to avoid infection is $1.85 .{ }^{1}$ However, one of the main problems in prescribing these antibiotics is increased bacterial resistance to antibiotics and the selection of multiresistant bacterial flora. Recent bibliographic reviews have shown 
that cranberry syrup may be useful in controlling recurrent UTI among healthy adults. ${ }^{1,2}$ Most of these studies have been aimed at subpopulations at greater risk of developing UTI, such as pregnant women or the elderly. In all cases, it has been concluded that, although the effect of cranberry syrup seems to be beneficial, its optimum dose remains unknown, as is its possible utility among a pediatric population, for which few studies have been made. UTI is relatively frequent in children, presenting a rate of around $8 \%$ among girls and $2 \%$ among boys. The recurrence of UTI following pyelonephritis may rise to $20 \% .^{3}$ Some later evidence ${ }^{3,4}$ seem to suggest that antibiotic prophylaxis does not affect the frequency of kidney scarring and so the utility of this treatment has been questioned. Other studies ${ }^{5}$ have shown that pediatric patients suffering from recurrent UTI and being treated with low-dose trimethoprim-sulfamethoxazole achieve a $6 \%$ reduction in the rate of UTI, in comparison with placebo treatment $(95 \%$ CI: $1-11)$. In previous studies, ${ }^{6}$ we confirmed the in vitro anti-adherent properties of the cranberry extract used in our assay of the adherent capacity of Escherichia coli. The present study, based on a controlled, double-blind clinical trial, examines the effectiveness of cranberry syrup treatment for an infant and children population at high risk of recurrent UTI. The study hypothesis is that cranberry syrup is safe and effective in the prophylaxis of urinary tract infection, equivalent or at least not inferior to trimethoprim for the prophylaxis of UTI.

\section{Methods}

\section{Study participants}

From 2009 to 2010, children aged from 1 month to 13 years, treated at the pediatric nephrology and urology departments in our hospital, were recruited for this study. The inclusion criteria established were a background of recurrent UTI (more than two episodes of infection in the last 6 months), vesicoureteral reflux of any degree, or renal pelvic dilatation associated with a UTI. The presence of infection was confirmed by urine culture $(>100,000$ colony forming units $[\mathrm{CFU}] / \mathrm{mL})$ using a 'mid-stream' urine sample or a urine collection bag, after asepsis of the urethral meatus with chlorhexidine, or $>10,000 \mathrm{CFU} / \mathrm{mL}$ if a catheter specimen of urine was obtained. In both cases, the presence or otherwise of a urine sediment with $>20$ leukocytes per field was determined. Criteria for exclusion from recruitment to the study included the co-existence of UTI with other infectious diseases or with metabolic diseases, chronic renal insufficiency, the presence of allergy or intolerance to any of the components of cranberry syrup or trimethoprim, the existence of blood dyscrasia, or the express wish of the parent or guardian to exclude the child from the study. This study was approved by the local ethics committee, and in all cases the parents or guardians gave their written informed consent.

\section{Primary and secondary outcomes}

Our clinical practice guidelines recommend trimethoprim for the prophylaxis of recurrent UTI in infants until an imaging study is performed and in all patients with vesicoureteral reflux (VUR) grade III and IV. The problems faced in our hospital regarding bacterial resistance to common antibiotics justify this comparative study, in which we seek evidence of positivity in order to reduce the consumption of antibiotics. We compare the efficacy and safety of cranberry prophylaxis in a single daily dose when either recurrent UTI or VUR, both of which are major indications for prophylaxis, is recognized. In the absence of references in this regard, and after approval by the ethics committee of our hospital, the study was extended to other diagnostic groups that are not the object of routine prophylaxis, namely dilatation of the renal pelvis, hydronephrosis, and renal anatomical defects.

The secondary outcome was the evaluation of the predominant bacterial flora in each arm of the intervention and of the most common bacterial resistance.

\section{Study design and intervention}

The study was designed as a Phase III randomized clinical trial, with the treatment option concealed from both patients and researchers. There were two treatment branches: cranberry syrup and trimethoprim. The study began on January 1, 2009 and ended on October 31, 2010. The maximum follow-up period of each patient recruited was 1 year.

\section{Randomization}

For the randomization, we used the SPSS macro published by Domenech et al (!RNDPB V2005.03.07 c), which randomized permuted blocks of five subjects in each of the centers. Each patient randomized to an intervention arm was assigned a registration number corresponding to a patient data file. The files were distributed sequentially in blocks of five among the recruitment points, as requested by the researchers when new patients were included in the study. The register numbers corresponded to those of the treatment interventions, and this list was only held by the Hospital Pharmacy Service.

After each patient was included in the study, the researcher gave the parents a card with the corresponding identification number. This card was presented at the Hospital Pharmacy 
Service to obtain the corresponding treatment, which was thus masked.

\section{Characterization of the cranberry syrup}

The cranberry syrup was supplied by Pharmatoka (Ruei Malmaison, France), using a dispersion of $2.8 \%$ of a high cranberry PAC (proanthocyanidin) extract used to formulate the food supplement Urell ${ }^{\circledR}$. The concentration guarantees that $5 \mathrm{~mL}$ of the syrup contains $36 \mathrm{mg}$ of highly bioactive PACs extracted from the cranberry syrup, measured by the BL-DMAC method. The cranberry syrup was characterized by the Department of Analytical Chemistry at the University of Granada. ${ }^{7}$

\section{Experimental group}

This group was given a nocturnal dose of $0.2 \mathrm{~mL} / \mathrm{kg}$ of cranberry syrup, just before the evening meal. If it was vomited during the following 30 minutes, administration of this dose was repeated.

\section{Control group}

This group was given a nocturnal dose of $0.2 \mathrm{~mL} / \mathrm{kg}$ of a color-masked suspension of trimethoprim at a concentration of $8 \mathrm{mg} / \mathrm{mL}$. This was given just before the evening meal, and if it was vomited during the following 30 minutes, administration of this dose was repeated.

\section{Masking}

At all times, the intention-to-treat principle was maintained. The trimethoprim syrup was diluted to a concentration of $8 \mathrm{mg} / \mathrm{mL}$. For the color masking, CC-1000-WS (E-120) colorant (CHR-Hansen) was added, at a concentration of $0.1 \%$ (not exceeding the $100 \mathrm{ppm}$ of carmine allowed). The packaging for both treatment options was mahogany-colored plastic tubes of $150 \mathrm{~mL}$. The tubes were labeled in accordance with the regulations of the Spanish Agency for Medicinal Products (AEM) for research medicines.

\section{Follow-up}

Treatment compliance was assessed by telephone consultation; every month, the parents were contacted to ask whether the child was taking the syrup and whether there were any problems in this respect. A periodic follow-up examination was performed, every 2 months or on demand from the patient when the symptoms called for it. The patient was instructed to obtain a urine culture if fever, urinary symptoms, vomiting, or weight loss occurred. The urine culture was also to be obtained before beginning any antibiotic treatment. All patients included in the study underwent a kidney ultrasound examination. The patients with renal pelvis dilatation and those with recurrent urinary tract infection were examined by serial voiding cystography. The patients with vesicoureteral reflux grade II or higher, or with moderate hydronephrosis, underwent renal scintigraphy. Data records were kept of the treatment number assigned, the clinical and analytical data for each of the patients' scheduled follow-up examinations, and the periodic results of the urine cultures obtained. The absence of fever was not considered as a criterion for exclusion.

\section{Sample size}

From our review of previous studies, ${ }^{3,8}$ there is assumed to be a $20 \%$ risk of recurrence of UTI during the first year that patients receive antibiotic treatment. In the present study, we examined the equivalence or otherwise of cranberry syrup prophylaxis and trimethoprim. The limit of equivalence was estimated at $\pm 10 \%$. The alpha error was estimated at $5 \%$ and the power of the study, at $80 \%$. In accordance with these data, the sample size was calculated to be 109 patients per group.

\section{Statistical analysis}

Statistical analysis was designed as a survival analysis in which appearance of the event (UTI) was sufficient cause for ending the follow-up period. In cases of voluntary withdrawal from the study or treatment discontinuation for reasons other than UTI, these data were considered to be censored for the remaining follow-up period. Kaplan-Meier curves were calculated for the experimental intervention and for the control treatment.

For the noninferiority analysis we consider a difference in prevalence $(\mathrm{d})$ of $0.105(18.9 \%-8.4 \%=10.5 \%)$,

$$
\begin{aligned}
& \mathrm{SE}=\sqrt{\frac{\text { Var cranberry }}{\mathrm{n} 1}+\frac{\text { Var trimethoprim }}{\mathrm{n} 2}} ; \\
& \mathrm{SE}=\sqrt{\frac{0.174}{37}+\frac{0.203}{58}}=0.09 .
\end{aligned}
$$

At baseline, the limit of equivalence was estimated at $\pm 0.1(10 \%) \rightarrow \delta^{\mathrm{L}}=-0.1, \delta^{\mathrm{U}}=0.1, \mathrm{z}_{\alpha}=1.96$.

We tested the noninferiority of cranberry versus trimethoprim. We raised only a unilateral noninferiority hypothesis:

$$
\begin{aligned}
\mathrm{H} 1_{\mathrm{L}} & =\mathrm{Pr}_{\text {cranberry }}-\mathrm{Pr}_{\text {trimethoprim }}>\delta^{\mathrm{L}} \rightarrow \mathrm{H} 0_{\mathrm{L}} \\
& =\mathrm{Pr}_{\text {cranberry }}-\mathrm{Pr}_{\text {trimethoprim }} \leq \delta^{\mathrm{L}}
\end{aligned}
$$

That is evidenced by the statistic $\mathrm{Z}_{\mathrm{L}}=\mathrm{d}-\delta^{\mathrm{L}} / \mathrm{SE}$; $\mathrm{Z}_{\mathrm{U}}=\mathrm{d}-\delta^{U} / \mathrm{SE}$. 


\section{Results}

Figure 1 shows the CONSORT flow diagram. A total of 198 eligible patients were included in the study. Gastrointestinal intolerance was observed in five of the cases assigned to trimethoprim and in two of those assigned to cranberry syrup treatment. An equal number of losses to follow-up were experienced in both branches of the study $(n=3)$; furthermore, in one case in each treatment branch, cutaneous rash was observed following administration. Urinary tract infection was observed in 47 patients, 17 of whom were males and 30 females. Thirty patients (14 given cranberry syrup and 16 given trimethoprim) completed the follow-up on a voluntary basis when their parents reported the absence of symptoms and made the unsupervised decision to discontinue prophylaxis (treatment discontinuation, Figure 1). Forty patients were less than 6 months of age. Of these, 24 (of whom eight received cranberry) completed the follow-up time (1 year) without a recurrence of UTI and without any adverse events. Two of the patients who received cranberry were less than 2 months of age.

Due to problems during the randomization process, 75 patients were assigned to receive cranberry syrup and 117 to receive trimethoprim. However, blinding to treatment was maintained. Table 1 shows the number $(\%)$ of patients with

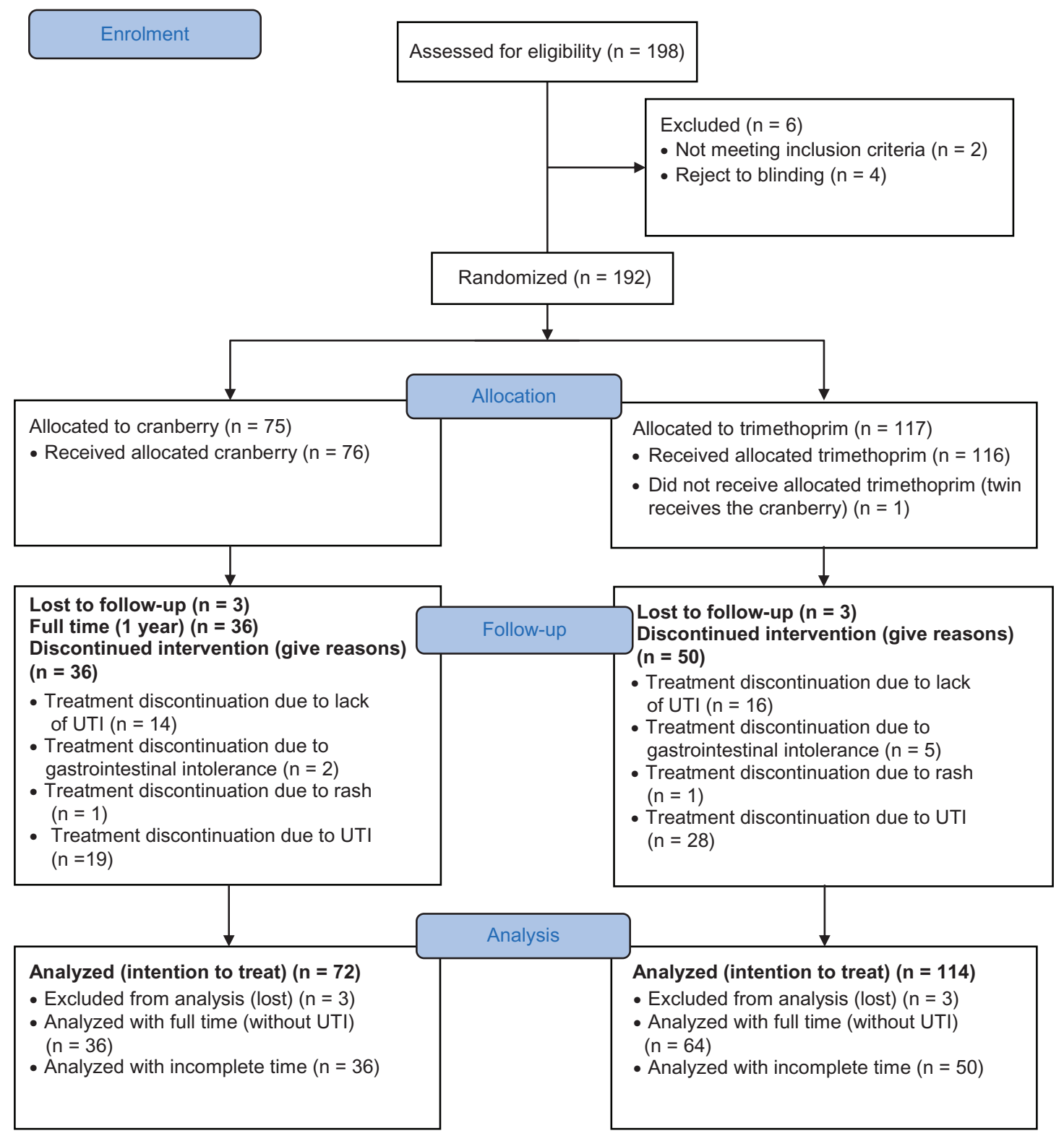

Figure I CONSORT 2010 flow diagram.

Note: Reproduced from CONSORT 2010 Statement; updated guidelines for reporting parallel group randomised trials: Schulz KF, Altman DG, Moher D. 340:c332. With permission from BMJ Publishing Group Ltd.

Abbreviation: UTI, urinary tract infection. 
Table I Number (\%) of patients with UTI in each treatment group

\begin{tabular}{|c|c|c|}
\hline & $\begin{array}{l}\text { Cranberry } \\
(n=75)\end{array}$ & $\begin{array}{l}\text { Trimethoprim } \\
(n=1 \mid 7)\end{array}$ \\
\hline Age, months (mean, SD) & $28.3(30.7)$ & $30.7(33.9)$ \\
\hline Gender, girls ( $\mathrm{n}$ ) & 43 & 69 \\
\hline \multicolumn{3}{|l|}{ Recurrent UTI } \\
\hline UTI, n (\%) & $8(8.4)$ & $18(18.9)$ \\
\hline Days without infection, mean (SD) & $229(128)$ & 207 (129) \\
\hline \multicolumn{3}{|l|}{ Vesicoureteral reflux } \\
\hline UTI, n (\%) & $5(12.8)$ & $4(10.3)$ \\
\hline Days without infection, mean (SD) & $240(139)$ & $281(119)$ \\
\hline \multicolumn{3}{|c|}{ Dilatation of renal pelvis or hydronephrosis } \\
\hline UTI, n (\%) & $5(9.8)$ & $5(9.8)$ \\
\hline Days without infection, mean (SD) & $192(120)$ & $207(128)$ \\
\hline \multicolumn{3}{|c|}{ Ureteral duplication and other anatomical defects } \\
\hline UTI, n (\%) & I (33.3) & I (33.3) \\
\hline Days without infection, mean (SD) & $29(13)$ & $159(137)$ \\
\hline
\end{tabular}

Note: Average time in days (SD) without infection in each treatment group. Abbreviation: UTI, urinary tract infection.

UTI in each treatment group, and states the average time in days (SD) without infection in each treatment group.

\section{Recurrent urinary tract infections}

Ninety-five of the patients were diagnosed with recurrent UTI on entry, and 26 of these suffered a UTI during followup (27.4\%, 95\% CI: $18.4 \%-36.3 \%)$. Six of these 95 (6.3\%) were male and $20(21.1 \%)$ were female. Eighteen of these patients $(18.9 \%, 95 \%$ CI: $11 \%-26.3 \%)$ received trimethoprim versus eight (8.4\%, $95 \%$ CI: $2.8 \%-13.9 \%$ ) who received cranberry.

As $\mathrm{z}_{\mathrm{U}}=0.23 \geq-\mathrm{z}_{\alpha}=-1.96$, we cannot accept the principle of equivalence between interventions, but the noninferiority of cranberry versus trimethoprim is shown.

Figure 2 shows the Kaplan-Meier curves for patients diagnosed on entry with recurrent UTI.

Of the patients recruited with recurrent UTI, the mean time to infection after taking trimethoprim was 271 days (95\% CI: 233-309 days) and after taking cranberry it was 312 days (95\% CI: 275-350 days). Logrank (Mantel-Cox) test: $2.14(P=0.14)$.

\section{Vesicoureteral reflux}

Thirty-nine of the patients recruited -19 males and 20 females - had VUR. Nine of those who completed the follow-up period presented UTI; four had been given trimethoprim $(10.3 \%, 95 \% \mathrm{CI}: 7.6 \%-19.8 \%)$ and five had received cranberry $(12.8 \%, 95 \%$ CI: $2.3 \%-23.2 \%)$. The small sample size of patients with VUR did not allow us to observe significant differences between the groups.
The mean time to infection after taking trimethoprim was 318 days (95\% CI: 274-362) and after taking cranberry it was 281 days (95\% CI: 317-344). Logrank (Mantel-Cox) test: $0.5(P=0.4)$.

\section{Dilatation of renal pelvis or hydronephrosis}

This group comprised 51 patients, of which 34 received trimethoprim and 17 cranberry prophylaxis. Of these patients, ten $(19.6 \%)$ developed UTI during follow-up, five (9.8\%) in each intervention arm. Of all infections observed in this group, seven (13.7\%) occurred in males and three $(5.9 \%)$ in females. The mean time to UTI after taking trimethoprim prophylaxis was 315 days (95\% CI: 275-354), while for those taking cranberry it was 270 days (95\% CI: 204-337). Logrank (Mantel-Cox) test: $1.33(P=0.24)$. No significant differences between interventions were observed.

\section{Ureteral duplication and other anatomical defects}

Seven of the patients recruited had these defects, and UTI was observed in two of them during follow-up.

Sixty-six of the episodes of UTI recurrence were caused by E. coli, with no significant differences being found between the two treatment branches. Table 2 shows the bacterial isolates in the urine of patients who completed follow-up. No differences between the treatment branches were observed in the rate of percentage of resistance to amoxicillin or cotrimoxazole $\left(\chi^{2}=2.7 ; P\right.$-value not significant and $\chi^{2}=0.3 ; P$-value not significant, respectively).

\section{Discussion}

Our study confirms that cranberry syrup is a safe treatment for the pediatric population, including infants aged less than 2 months. Cranberry prophylaxis is not equivalent to trimethoprim, but it is shown to be noninferior versus trimethoprim in recurrent UTI. Recent placebo-controlled clinical trials ${ }^{5}$ have shown the benefits of trimethoprimsulfamethoxazole for prophylaxis of recurrent UTI.

Previous studies, ${ }^{9,10}$ have reported the beneficial effect of cranberry syrup in preventing UTI among women, achieving a reduction in the absolute risk of UTI infection with respect to placebo treatment. This effect has been explained in terms of the anti-adherent effect of cranberry on E. coli. ${ }^{11}$ Ferrara et al ${ }^{12}$ in a controlled clinical trial with respect to placebo treatment in children aged over 3 years, showed that cranberry syrup prevents the recurrence of symptomatic UTI. 


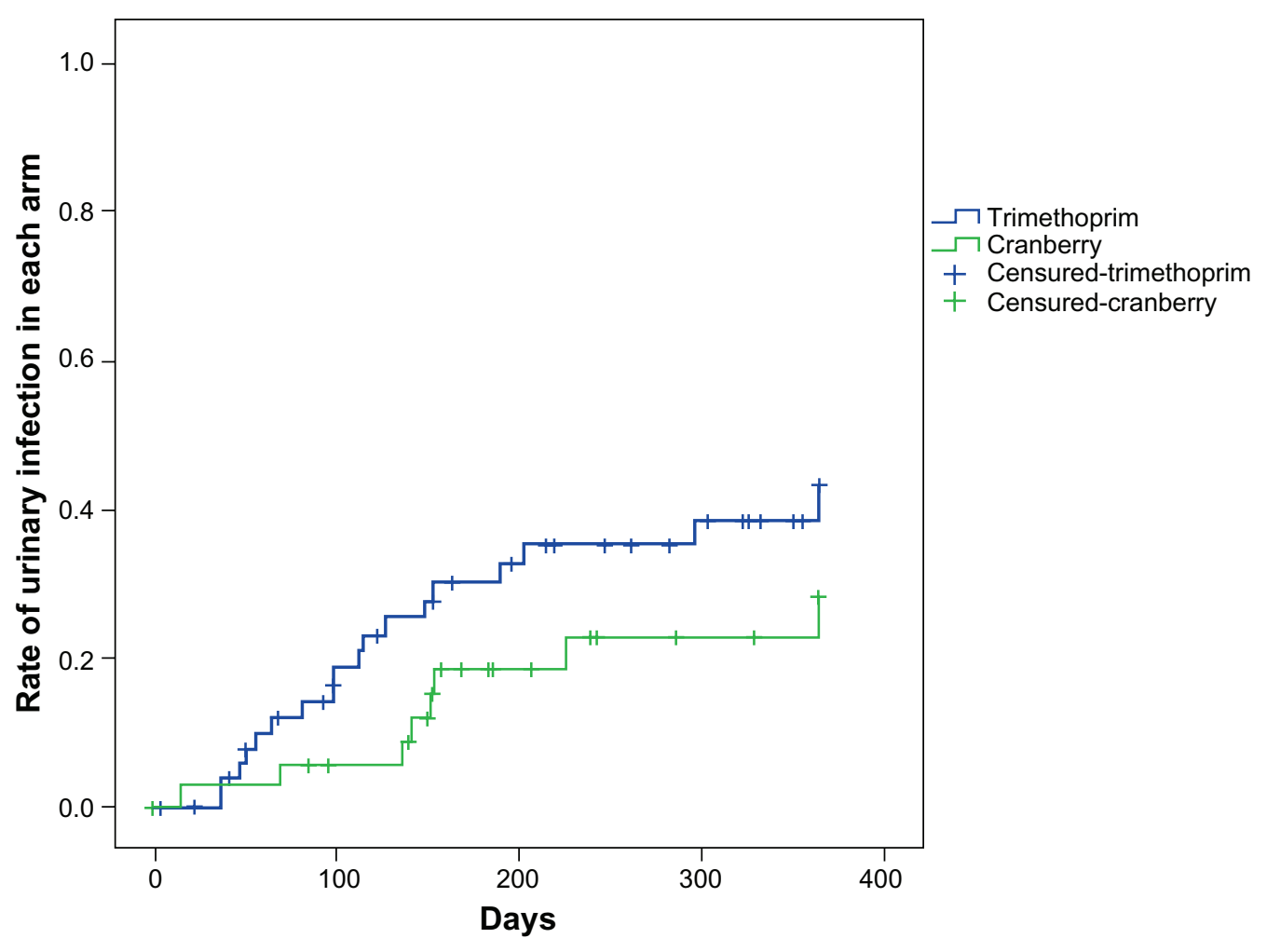

Figure 2 Kaplan-Meier curves for patients diagnosed with recurrent urinary infection on entry.

Under the principles of the Declaration of Helsinki, a clinical trial with placebo cannot be carried out in our circumstances,${ }^{13}$ and therefore, for ethical reasons, the present study was designed as a test of equivalence or non-inferiority. Clinical practice guidelines recommend the use of trimethoprim prophylaxis in children with VUR grade III and IV. In addition, some trials such as Craig et $\mathrm{al}^{5}$ have observed benefits from antibiotic prophylaxis in pediatric recurrent UTI.

Foo et al $^{14}$ identified trimeric proanthocyanidins and type A dimeric procyanidins as the factors responsible for the anti-adherent effect of cranberry. The composition of cranberry syrup used in our study could be of interest for future comparison of our results with those reported in other studies, given the great variability in the concentrations of proanthocyanidin observed in different cranberry extracts. ${ }^{15,16}$ Howell et a ${ }^{17}$ reported that the administration of cranberry syrup containing the equivalent of $72 \mathrm{mg}$ per day of proanthocyanidin has an anti-adherent effect, measured in urine, against $E$. coli that is proportionally superior to that obtained by patients given 18 or $36 \mathrm{mg}$. Di Martino et a ${ }^{11}$ tested the anti-adherent effect in urine of cranberry syrup administered to ten healthy volunteers, in relation to ten volunteers who were given placebo treatment.

Salo et a ${ }^{18}$ published a double-blind trial comparing cranberry juice with placebo in children with recurrent UTI, and observed that the intervention did not reduce the number of urinary infections. Unlike our cranberry syrup, which was characterized, ${ }^{7}$ the cranberry juice used in the above study had a nonstandardized concentration of polyphenols. We believe

Table 2 Bacterial isolates in the urine of patients who completed follow-up

\begin{tabular}{|c|c|c|c|c|}
\hline & \multicolumn{2}{|c|}{ Trimethoprim prophylaxis } & \multicolumn{2}{|l|}{ Cranberry prophylaxis } \\
\hline & Amoxicillin resistance & $\overline{\text { Cotrimoxazole resistance }}$ & Amoxicillin resistance & $\overline{\text { Cotrimoxazole resistance }}$ \\
\hline E. coli & 12 & 8 & 10 & 5 \\
\hline Proteus & 3 & I & 2 & I \\
\hline Klebsiella & I & 0 & 3 & 0 \\
\hline Staphylococcus & I & 0 & 0 & - \\
\hline Enterococcus & I & 0 & 0 & - \\
\hline Enterobacter & I & I & 0 & - \\
\hline
\end{tabular}


it is essential, in this type of study, to characterize the extracts used and to normalize the contents of the active polyphenolic fractions of each extract.

Two studies have evaluated the effectiveness of cranberry syrup for children with neurogenic bladder, monitored by means of a sterile intermittent catheter. ${ }^{19,20}$ The first of these studies had a simple cross-sectional design and included 40 children, while the second was randomized and placebo controlled, and included 15 children. Both studies concluded that cranberry syrup is no more effective than placebo treatment for controlling UTI among patients with neurogenic bladder. However, in neither case was the polyphenol content of the syrups characterized.

McMurdo et $\mathrm{al}^{21}$ carried out a randomized, double-blind clinical trial with cranberry or trimethoprim for women at risk of recurrent UTI. These authors found the risk of recurrence to be $60 \%$ greater among the patients who were given cranberry syrup, although the difference was not statistically significant. The systematic review published by Jepson et $\mathrm{al}^{1}$ of a total of ten studies (1049 patients) concluded that cranberry syrup significantly reduced the incidence of UTI at 12 months (OR: 0.65; 95\% CI: 0.46-0.90) in comparison with placebo/control treatments. Cranberry was more effective in reducing the incidence of UTI in women with recurrent infection than in men and elderly women, or persons requiring catheterization.

It was drawn to our attention after the trial that 75 patients received prophylaxis with cranberry and 117 with trimethoprim. This difference in the sizes of the treatment arms was due to an unfortunate problem with the randomization of patients, due to the misfiling of randomization folders in some medical practices. We believe these errors, which occurred toward the end of the study, may represent a bias in the randomization, but not a loss of masking, since the researchers did not know which treatment corresponded to each file. In any case, we observed no difference in the infection survival times in patients receiving cranberry with respect to those receiving trimethoprim, despite the lower number of patients in the cranberry treatment branch.

The prophylaxis of UTI has been associated with increased bacterial resistance to antibiotics. ${ }^{22}$ In our sample, we did not observe a significant increase in this respect between the two branches of the trial.

Our results show the noninferiority of cranberry syrup versus trimethoprim in the prophylaxis of recurrent urinary tract infections. Cranberry syrup is a safe treatment for the pediatric population and may constitute a valid alternative approach, especially in view of increasing resistance to antibiotics.

\section{Acknowledgments}

This clinical trial was funded by the Carlos III Institute of Health for Clinical Research, Madrid, Spain.

\section{Disclosure}

The authors report no conflicts of interest in this work.

\section{References}

1. Jepson RG, Mihaljevic L, Craig J. Cranberries for preventing urinary tract infections. Cochrane Database Syst Rev. 2004;2:CD001321.

2. Cimolai N, Cimolai T. The cranberry and the urinary tract. Eur J Clin Microbiol Infect Dis. 2007;26(11):767-776.

3. Garin EH, Olavarria F, Garcia NV, Valenciano B, Campos A, Young L. Clinical significance of primary vesicoureteral reflux and urinary antibiotic prophylaxis after acute pyelonephritis: a multicenter, randomized, controlled study. Pediatrics. 2006;117(3):626-632.

4. Mathews R, Carpenter M, Chesney R, et al. Controversies in the management of vesicoureteral reflux: the rationale for the RIVUR study. J Pediatr Urol. 2009;5(5):336-341.

5. Craig JC, Simpson JM, Williams GJ, et al; Prevention of Recurrent Urinary Tract Infection in Children with Vesicoureteric Reflux and Normal Renal Tracts (PRIVENT) Investigators. Antibiotic prophylaxis and recurrent urinary tract infection in children. $N$ Engl $J$ Med. 2009;361(18):1748-1759.

6. Uberos J, Iswaldi I, Rodríguez-Belmonte R, et al. Cranberry (Vaccinium macrocarpon) changes the surface hydrophobicity and biofilm formation of E. coli. Microbiology Insights. 2011;4:21-27.

7. Iswaldi I, Gomez-Caravaca AM, Arraez-Roman D, et al. Characterization by high-performance liquid chromatography with diode-array detection coupled to time-of-flight mass spectrometry of the phenolic fraction in a cranberry syrup used to prevent urinary tract diseases, together with a study of its antibacterial activity. $J$ Pharm Biomed Anal. 2012;58:34-41.

8. Savage DC, Howie G, Adler K, Wilson MI. Controlled trial of therapy in covert bacteriuria of childhood. Lancet. 1975;1(7903):358-361.

9. Kontiokari T, Sundqvist K, Nuutinen M, Pokka T, Koskela M, Uhari M. Randomised trial of cranberry-lingonberry juice and Lactobacillus GG drink for the prevention of urinary tract infections in women. BMJ. 2001;322(7302):1571.

10. Kontiokari T, Salo J, Eerola E, Uhari M. Cranberry juice and bacterial colonization in children - a placebo-controlled randomized trial. Clin Nutr. 2005;24(6):1065-1072.

11. Di Martino P, Agniel R, David K, et al. Reduction of Escherichia coli adherence to uroepithelial bladder cells after consumption of cranberry juice: a double-blind randomized placebo-controlled cross-over trial. World J Urol. 2006;24(1):21-27.

12. Ferrara P, Romaniello L, Vitelli O, Gatto A, Serva M, Cataldi L. Cranberry juice for the prevention of recurrent urinary tract infections: A randomized controlled trial in children. Scand J Urol Nephrol. 2009;43(5):369-372.

13. Braga LHP, Bagli DJ, Lorenzo AJ. Placebo-controlled trials in pediatric urology: A cautionary view from an ethical perspective. J Pediatr Urol. 2010;6(5):435-442.

14. Foo LY, Lu Y, Howell AB, Vorsa N. A-Type proanthocyanidin trimers from cranberry that inhibit adherence of uropathogenic P-fimbriated Escherichia coli. J Nat Prod. 2000;63(9):1225-1228.

15. Latti AK, Kainulainen PS, Hayirlioglu-Ayaz S, Ayaz FA, Riihinen KR. Characterization of anthocyanins in caucasian blueberries (Vaccinium arctostaphylos L) native to Turkey. J Agric Food Chem. 2009;57(12):5244-5249. 
16. Foo LY, Lu Y, Howell AB, Vorsa N. The structure of cranberry proanthocyanidins which inhibit adherence of uropathogenic P-fimbriated Escherichia coli in vitro. Phytochemistry. 2000;54(2):173-181.

17. Howell AB, Botto H, Combescure C, et al. Dosage effect on uropathogenic Escherichia coli anti-adhesion activity in urine following consumption of cranberry powder standardized for proanthocyanidin content: a multicentric randomized double blind study. BMC Infect Dis. 2010;10:94.

18. Salo J, Uhari M, Helminen M, et al. Cranberry Juice for the Prevention of Recurrences of Urinary Tract Infections in Children: A Randomized Placebo-Controlled Trial. Clin Infect Dis. 2012;54(3):340-346.

19. Foda MM, Middlebrook PF, Gatfield CT, Potvin G, Wells G, Schillinger JF. Efficacy of cranberry in prevention of urinary tract infection in a susceptible pediatric population. Can J Urol. 1995;2(1): 98-102.
20. Schlager TA, Anderson S, Trudell J, Hendley JO. Effect of cranberry juice on bacteriuria in children with neurogenic bladder receiving intermittent catheterization. J Pediatr. 1999;135(6):698-702.

21. McMurdo ME, Argo I, Phillips G, Daly F, Davey P. Cranberry or trimethoprim for the prevention of recurrent urinary tract infections? A randomized controlled trial in older women. JAntimicrob Chemother. 2009;63(2):389-395.

22. Cheng $\mathrm{CH}$, Tsai $\mathrm{MH}$, Huang $\mathrm{YC}$, et al. Antibiotic resistance patterns of community-acquired urinary tract infections in children with vesicoureteral reflux receiving prophylactic antibiotic therapy. Pediatrics. 2008;122(6):1212-1217.

\section{Publish your work in this journal}

The Open Access Journal of Clinical Trials is an international, peerreviewed, open access journal publishing original research, reports, editorials, reviews and commentaries on all aspects of clinical trial design, management, legal, ethical and regulatory issues, case record form design, data collection, quality assurance and data auditing methodologies. The manuscript management system is completely online and includes a very quick and fair peer-review system, which is all easy to use. Visit http://www.dovepress.com/testimonials.php to read real quotes from published authors.

Submit your manuscript here: http://www.dovepress.com/open-access-journal-of-clinical-trials-journal 